\title{
Runx3 expression in lymph nodes with metastasis is associated with the outcome of gastric cancer patients
}

\author{
HONG-WEI XU ${ }^{1}$, FENG REN ${ }^{2}$, YAN-MING YU ${ }^{3}$ and CHENG-ZHONG CAI ${ }^{4}$ \\ Departments of ${ }^{1}$ General Surgery, and ${ }^{2}$ Clinical Laboratory, the Fourth Affiliated Hospital of Soochow University, \\ Wuxi, Jiangsu 214062; ${ }^{3}$ Huangpu Central Hospital of Shanghai, Shanghai 200002; \\ ${ }^{4}$ The Tenth People's Hospital of Shanghai Tongji University, Shanghai 200072, P.R. China
}

Received April 1, 2011; Accepted August 2, 2011

DOI: $10.3892 / \mathrm{ol} .2011 .381$

\begin{abstract}
Accumulating evidence shows that runt-related transcription factor 3 (Runx3) is a putative tumor suppressor in various types of cancer, the lower levels of which are associated with a less favorable cancer outcome. However, these studies were restricted to primary cancer lesions. Lymph node metastasis (LNM) is a significant factor in determining the prognosis of patients with gastric cancer and is a frequent target of chemotherapy. In the present study, we investigated the expression of Runx3 in the lymph nodes (LNs) of stomach carcinoma and the association of Runx3 expression with the prognosis of patients. The expression of Runx 3 in LNs with and without metastasis was detected by reverse transcriptase-polymerase chain reaction (RT-PCR) and Western blotting. The positive rate of Runx3 mRNA in LNM specimens was significantly lower $(28.4 \%, 21$ out of 74$)$ compared to that of the non-metastatic samples $(33.3 \%, 9$ out of 27, $\mathrm{P}<0.05)$. Similar findings were obtained by Western blotting. Univariate analysis revealed that the loss of Runx3 expression in LNs was not only associated with poor clinicopathological factors, such as LNM, distant organ metastasis, later clinicopathological stages and deep infiltration, but also with a lower 5-year survival rate and poorer prognosis. These results strongly suggest a potential diagnostic value of Runx3 expression in LNs and multiple pathways contributing to the outcome of patients with gastric cancer.
\end{abstract}

\section{Introduction}

Gastric cancer remains a major health problem and a leading cause of cancer-related death, although its incidence has decreased worldwide (1). Numerous patients are identified for gastric cancers at the advanced stage, which is associated with

Correspondence to: Dr Hong-Wei Xu, Department of General Surgery, the Fourth Affiliated Hospital of Soochow University, 200 Huihe Road, Wuxi, Jiangsu 214062, P.R. China

E-mail: xuhongwei@ymail.com

Key words: stomach neoplasms, lymph node metastasis, Runx3, prognosis increased recurrence and low overall survival (OS) following potentially curative resection (2). Radical surgery, including lymph node ( $\mathrm{LN}$ ) dissection, has been the standard treatment for early gastric cancer; however, $50 \%$ of gastric cancer patients suffer from tumor relapses even after radical surgery $(3,4)$, and their overall prognosis is suboptimal despite aggressive treatment. In recent years, much evidence has clearly demonstrated that multiple genetic changes are responsible for the development and progression of gastric cancer. Thus, it is imperative to investigate the molecular mechanism to improve the outcome of patients with gastric cancer.

Studies have demonstrated that the aggressive nature of gastric cancer is related to mutations of various oncogenes and tumor suppressor genes, as well as abnormalities in certain growth factors and their receptors (5). Recent studies have shown that runt-related transcription factor 3 (Runx3) gene mutation is significantly associated with primary gastric cancer progression. However, the detailed mechanism for this relationship has yet to be completely clarified (6). The Runx3 gene encodes a protein that belongs to the runt domain family of transcription factors involved in mammalian development pathways (7). Runx3 protein mediates the growth suppression effects of TGF- $\beta$ in association with SMAD, a downstream protein in the signaling pathway $(8,9)$. Previous studies demonstrated that Runx3 is markedly down-regulated in gastric cancers compared to the surrounding mucosa, and that lack of Runx 3 is causally related to the growth and progression of human gastric cancer (10), indicating that Runx3 is a novel tumor suppressor (11-14).

It is known that the down-regulation of Runx3 in primary gastric cancer tissues is associated with poor prognosis. However, less is known about its expression in LNs, particularly the relationship between the expression of Runx3 and the prognosis of patients. Lymph node metastasis (LNM) is a significant factor for determining the prognosis of patients with gastric cancer and is a frequent target for chemotherapy. Therefore, it is essential to analyze Runx3 gene expression in LNs from gastric cancer. In this study, reverse transcriptasepolymerase chain reaction (RT-PCR) and Western blotting were used to assess the expression level of Runx3 in 101 LNs of stomach carcinoma, and to describe for the first time the association between the expression of Runx3 gene in LNs and the outcome of patients with gastric cancer. 


\section{Materials and methods}

Tissue samples. LN specimens were obtained from 101 patients who were diagnosed with primary gastric cancer and underwent gastrectomy and LN dissection in the Department of Surgery, the Tenth People's Hospital of Shanghai, Tongji University, China, between October 2000 and October 2002. The samples were rapidly frozen in liquid nitrogen and stored at $-80^{\circ} \mathrm{C}$ until being used for the extraction of RNA and protein. The gastric cancer patients had well-documented clinical histories and follow-up information. Clinicopathological data were obtained from a retrospectively constructed medical database, which had been reviewed and confirmed by two pathologists. Patients who had been preoperatively treated with radiation and/or chemotherapy were excluded. Informed consent was obtained from each of the patients and the study protocol was approved by the Ethics Committee of the Tenth People's Hospital of Shanghai, China. Details of the patient characteristics and Runx3 expression are provided in Table I. Following radical gastrectomy, patients were followed up until death or October 31, 2009, as appropriate. The median follow-up duration was 35.6 months. At the last follow-up examination, $22(21.8 \%)$ patients were still alive, whereas 79 (78.2\%) patients had succumbed.

RNA extraction and RT-PCR. LN tissues were homogenized with an ultrasound homogenizer. Total RNA was extracted using TRIzol reagent (Invitrogen, Carlsbad, CA, USA) according to the manufacturer's instructions. Total RNA $(1 \mu \mathrm{g})$ was reversely transcribed into cDNA using dNTPs $(1 \mathrm{mM})$, $5 \mathrm{X}$ reverse transcription buffer $(500 \mathrm{mM}$ Tris-HCL pH 8.3, $250 \mathrm{mM} \mathrm{KCL}, 50 \mathrm{mM} \mathrm{MgCl}$ and $50 \mathrm{mM}$ DTT), 16 units RNasin and 2.5 units AMV reverse transcriptase (Gibco BRL, Life Technologies, Carlsbad, CA, USA). PCR conditions were: pre-heating at $95^{\circ} \mathrm{C}$ for $5 \mathrm{~min}$ followed by 35 cycles of denaturation for $30 \mathrm{sec}$ at $95^{\circ} \mathrm{C}$, annealing for $1 \mathrm{~min}$ at $55^{\circ} \mathrm{C}$ and extension for $1 \mathrm{~min}$ at $72^{\circ} \mathrm{C}$, with a final extension for $5 \mathrm{~min}$ at $72^{\circ} \mathrm{C}$. PCR products were separated on $1.5 \%$ agarose gel and saved as digital images (Perkin-Elmer, Wellesley, MA, USA). These experiments were performed in triplicate and the mean value was calculated. The value was normalized as the target gene divided by $\beta$-actin. The primers used were: Runx 3 gene, forward 5'-ATGACGAGAACTACTCCGCT-3' and reverse 5'-GGTCGGAGAATGGGTTCAGT-3' (PCR product, 396 bp).

Western blotting. The LN homogenates were heated in boiling water for $5 \mathrm{~min}$. Protein concentrations were measured using Bradford's method (Bio-Rad, Hercules, CA, USA). Protein $(50 \mu \mathrm{g})$ was loaded, separated by $10 \%$ SDS-polyacrylamide gel electrophoresis under reducing conditions, and transferred onto equilibrated polyvinylidene difluoride membrane (PVDF; Amersham) by electronic transfer. Membranes were blocked by $5 \%$ non-fat dried milk and then incubated with an antibody against Runx3 (dilution 1:200) overnight at $4^{\circ} \mathrm{C}$; incubation with the secondary antibody was $1 \mathrm{~h}$ at room temperature, with three washes after each incubation. ECL reagents were used to show the positive bands on the membrane.

Statistical analysis. Statistical analysis was performed using SPSS 17.0 software. Continuous variables were determined as the means \pm SD and compared using the two-tailed version
Table I. Association between the expression of Runx3 and clinicopathological characteristics of patients with gastric cancer.

\begin{tabular}{|c|c|c|c|c|}
\hline \multirow{2}{*}{$\begin{array}{l}\text { Clinicopathological } \\
\text { parameters }\end{array}$} & \multirow[t]{2}{*}{ No. } & \multicolumn{2}{|c|}{ Runx3 mRNA } & \multirow[t]{2}{*}{ P-value } \\
\hline & & $\leq 0.362$ & $>0.362$ & \\
\hline Gender & & & & $>0.050$ \\
\hline Male & 69 & 38 & 31 & \\
\hline Female & 32 & 12 & 20 & \\
\hline Age (years) & & & & $>0.050$ \\
\hline$<60$ & 46 & 21 & 25 & \\
\hline$\geq 60$ & 55 & 29 & 29 & \\
\hline Growth pattern & & & & $<0.050$ \\
\hline Expansive & 39 & 24 & 15 & \\
\hline Infiltrative & 62 & 41 & 21 & \\
\hline Histological grade & & & & $>0.050$ \\
\hline WD and MD & 55 & 31 & 24 & \\
\hline PD & 46 & 20 & 26 & \\
\hline Infiltrative depth & & & & $<0.050$ \\
\hline $\mathrm{T} 1+\mathrm{T} 2$ & 44 & 29 & 15 & \\
\hline $\mathrm{T} 3+\mathrm{T} 4$ & 56 & 32 & 24 & \\
\hline LN metastasis & & & & $<0.001$ \\
\hline Absence & 27 & 18 & 9 & \\
\hline Presence & 74 & 53 & 21 & \\
\hline Distant metastasis & & & & $<0.001$ \\
\hline Absence & 73 & 42 & 31 & \\
\hline Presence & 28 & 17 & 11 & \\
\hline TNM stage & & & & $<0.001$ \\
\hline I and II & 24 & 19 & 5 & \\
\hline III and IV & 77 & 55 & 22 & \\
\hline
\end{tabular}

LN, lymph node; WD, well-differentiated; MD, moderately differentiated; PD, poorly differentiated.

of Fisher's exact test. The correlation was evaluated using regression analysis. Survival was analyzed by the KaplanMeier method, and differences in the distribution were evaluated using the log-rank test. $\mathrm{P}<0.05$ was considered to be statistically significant.

\section{Results}

Expression of Runx3 in LN specimens. As shown in Fig. 1, Runx 3 mRNA was examined in 74 LNs with metastasis and 27 LNs without metastasis. RT-PCR results revealed that Runx 3 was positively expressed in $28.4 \%$ (21 out of 74) LNs with metastasis and $33.3 \%$ (9 out of 27) without metastasis $(\mathrm{P}<0.001)$. The expression of Runx3 protein in LNs was further confirmed by immunoblotting, which revealed a 44-kDa protein band (Fig. 2). Similarly, the results also revealed that expression of the Runx3 protein was significantly lower in LNM tissues than in those without metastasis. A consistent correlation was found between the protein and mRNA expression of Runx3. 
A

bp MarkerM1 N1 M2 N2 M3 N3

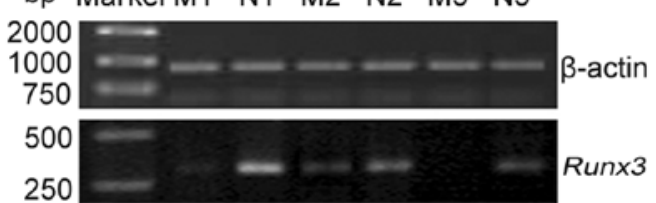

B

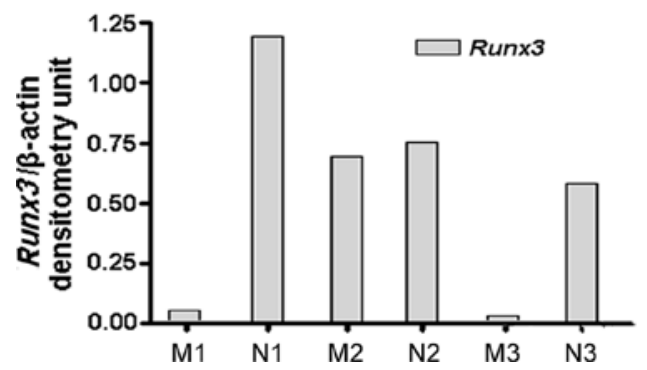

Figure 1. (A) Expression of Runx3 mRNA in LN specimens with metastasis (M) and without metastasis (N). The 396-bp human Runx3-specific sequence and a 906-bp $\beta$-actin sequence were amplified from the cDNA of gastric cancer LN tissues, separated by agarose gel electrophoresis and visualized by ethidium bromide staining. (B) Densitometry of Runx3 transcripts standardized to $\beta$-actin, for the conditions listed in (A).
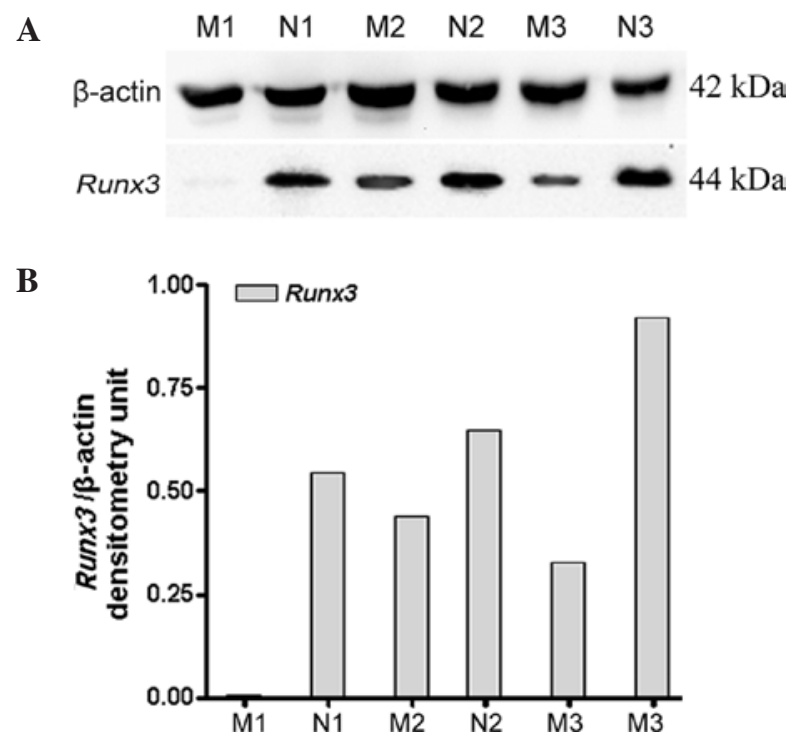

Figure 2. (A) Representative samples of Western blotting Runx3 proteins in human gastric cancer LNs, LN specimens with metastasis (M) and without metastasis $(\mathrm{N})$. The upper panel shows the $\beta$-actin levels as the internal control. (B) Densitometry of Runx3 proteins standardized to $\beta$-actin, for the conditions listed in (A).

Relationship between the expression levels of Runx3 and clinicopathological parameters. The clinicopathological characteristics of the patients and associations with Runx3 mRNA expression in LNs are shown in Table I. The gastric cancer patients (69 males and 32 females with a median age of 68.6 years; range 45-88) had undergone gastrectomy and lymphadenectomy. Seventy-four patients were diagnosed with LNM. Thirty-nine patients had an expansive growth pattern, whereas 62 had an infiltrative classification. With respect to TNM tumor staging, gastric cancer LNs with a higher expression of Runx3 were $20.8 \%$ (5 out of 24) at stages I and II, and $28.6 \%$ (22 out of 77 ) at stages III and IV, respectively. Univariate analysis showed that the level of Runx3 mRNA

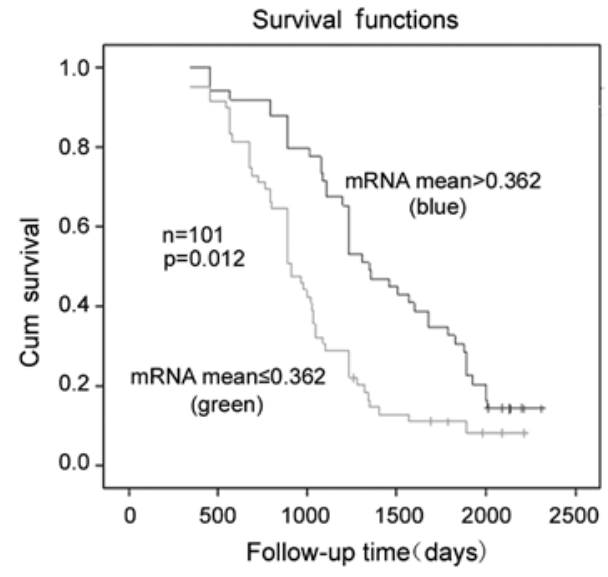

Figure 3. Kaplan-Meier survival analysis for gastric cancer patients according to the expression of Runx3. The y-axis shows the percentage of patients; the $\mathrm{x}$-axis, their survival in days. The green line shows LN specimens with a lower $(\leq 0.362)$ Runx3 mRNA expression, with a trend of poorer survival than the blue line, indicating specimens with a higher $(>0.362)$ Runx3 mRNA expression. Expression in LN specimens (log-rank test, $\mathrm{P}=0.012$ ). Mean survival time was 1,021 days for the lower expression group and 1,234 days for the higher expression group.

expression was inversely correlated with advanced clinical stage $(\mathrm{P}<0.001)$, distant metastasis $(\mathrm{P}<0.05)$, LNM $(\mathrm{P}<0.001)$, infiltrative depth $(\mathrm{P}<0.05)$ and histological grade $(\mathrm{P}<0.001)$. However, no significant associations were observed with patient age or gender $(\mathrm{P}>0.05)$, or with the growth pattern of the tumor $(\mathrm{P}>0.05)$ (Table I).

Survival analysis. The patients had a median follow-up duration of 35.6 months (range 3-84) following radical gastrectomy. Seventy-nine patients succumbed due to recurrence or metastasis of the tumor and 22 patients survived. According to the median of the gray scale bands $(0.362$; range 0.088 1.016) for Runx3 mRNA expression in LNs, the 101 cases were separated into two groups: the lower-expressing group $(\leq 0.362)$ and the over-expressing group $(>0.362)$. KaplanMeier analysis confirmed that patients with a higher Runx3 expression $(>0.362)$ had significantly better survival outcomes compared to the lower group $(\leq 0.362)$. The median OS for the high and low Runx3 mRNA expression groups were 1,234 and 1,021 days, with a 5-year survival rate of 16 and $28 \%$, respectively (log-rank test, $\mathrm{P}=0.012$; Fig. 3).

\section{Discussion}

In the present study, Runx3 expression was first examined at the RNA and protein levels in the LN tissues of gastric cancer using RT-PCR and Western blotting. The results indicate that the expression of Runx 3 mRNA was more frequent in the LNs without metastasis than in the metastatic regions. Western blotting demonstrated similar findings. Loss of Runx3 expression was associated with a lower 5-year survival rate and poorer prognosis of patients.

Runx3 is a transcription factor that regulates lineagespecific gene expression in developmental processes and is involved in the formation of a variety of cancers. Runx3 elicits its tumor suppressor role by controlling the expression of numerous genes involved in the growth, apoptosis and 
differentiation of gastric epithelial cells (15-19), as well as genes involved in angiogenesis and cell junction formation $(20,21)$. Mounting evidence suggests that Runx3 is a tumor suppressor. Runx 3 is expressed in glandular stomach epithelial cells, and loss of Runx 3 expression is causally related to the genesis and progression of gastric cancer and correlates with differentiation, metastasis and poor prognosis of gastric cancer (22).

In primary gastric cancer, much is known about the expression of Runx3, but less is known about the relationship between Runx3 expression in LNs and the prognosis of patients. In this study, various clinicopathological factors were analyzed for Runx3 expression in LNs. A low proportion of Runx 3 mRNA expression in LNM was found to be significantly associated with poor clinicopathological factors, such as deep infiltration, distant organ metastasis, poor differentiation, LNM and later clinicopathological stages. However, no significant associations were observed with age, gender and the growth pattern of the tumor (Table I). Runx3 expression was also associated with a lower 5-year survival rate and poorer prognosis of patients (Fig. 3). These results support our previous studies (23), which revealed that the low expression of Runx3 in primary gastric cancer was associated with a significantly shorter survival.

LNM is one of the most significant factors predicting recurrence in patients who have undergone gastrectomy for stomach carcinoma $(24,25)$. In China, a gastrectomy along with an extended LN dissection is an established procedure and widely accepted as the standard for the surgical treatment of gastric cancer. However, certain patients develop local or distant tumor recurrence even if a curative resection of the primary tumor is performed. In our studies, the rate of LNM in gastric cancer was $73.7 \%$, which is higher than that previously reported in Japan and similar to reports from China $(26,27)$. This finding may be explained due to the bias of histological criteria employed in our study and those used in Japan. We also found that the presence of metastasis is significantly correlated with the postoperative prognosis of patients with gastric cancer (data not shown).

Concerning LNM, Park et al (28) reported MGMT expression to be significantly associated with LNM in patients with gastric cancer. Gene analysis along with a protein examination is recommended to increase the positive detection of LNM and prognosis, leading to a more accurate diagnosis and therapy of the tumor in gastric cancer. Furthermore, such an analysis may positively contribute to the selection of optimal chemotherapeutic regimens based on the gene. Compared to the data generated in this study, Runx 3 gene expression in the LNs was significantly associated with poor clinicopathological factors and poorer prognosis of patients. Consequently, a gene expression analysis using $\mathrm{LN}$ tissue specimens may aid in the detection of the survival rate and prognosis of stomach carcinoma. However, the precise link between Runx3 expression in LNs and LNM remains unclear and further biological studies are required to explain this effect (29).

In conclusion, a loss or substantial decrease of Runx3 expression was observed in the group presenting with $\mathrm{LN}$ with metastasis as compared to the group without metastasis, and a low expression of Runx 3 was significantly associated with unfavorable clinicopathological variables and a shorter survival in gastric carcinoma patients. LNM is also associated with a poorer prognosis of gastric cancer. It may contribute to the detection of gene expression and gastric cancer metastasis as a potential molecular marker and a potential target in the therapy for gastric cancer.

\section{Acknowledgements}

This study was supported by grants from the National Natural Science Foundation of China (no. 30171039) and the Natural Science Foundation of Jiangsu Province (no. BK2008115).

\section{References}

1. Kamangar F, Dores GM and Anderson WF: Patterns of cancer incidence, mortality, and prevalence across five continents: defining priorities to reduce cancer disparities in different geographic regions of the world. J Clin Oncol 24: 2137-2150, 2006.

2. Rohatgi PR, Yao JC, Hess K, et al: Outcome of gastric cancer patients after successful gastrectomy: influence of the type of recurrence and histology on survival. Cancer 107: 2576-2580, 2006.

3. Nashimoto A, Nakajima T, Furukawa $\mathrm{H}$, et al: Randomized trial of adjuvant chemotherapy with mitomycin, Fluorouracil, and Cytosine arabinoside followed by oral Fluorouracil in serosanegative gastric cancer: Japan Clinical Oncology Group 9206-1. J Clin Oncol 21: 2282-2287, 2003.

4. Marrelli D, De Stefano A, de Manzoni G, Morgagni P, Di Leo A and Roviello F: Prediction of recurrence after radical surgery for gastric cancer: a scoring system obtained from a prospective multicenter study. Ann Surg 241: 247-255, 2005.

5. Deng JY, Sun D, Liu XY, Pan Y and Liang H: STAT-3 correlates with lymph node metastasis and cell survival in gastric cancer. World J Gastroenterol 16: 5380-5387, 2010.

6. Tsang YH, Lamb A, Romero-Gallo J, et al: Helicobacter pylori CagA targets gastric tumor suppressor RUNX3 for proteasomemediated degradation. Oncogene 29: 5643-5650, 2010.

7. Ito Y: Runx genes in development and cancer: regulation of viral gene expression and the discovery of Runx family genes. Adv Cancer Res 99: 33-76, 2008.

8. Ito K, Lim AC, Salto-Tellez M, et al: Runx3 attenuates $\beta$-catenin/T cell factors in intestinal tumourigenesis. Cancer Cell 14: 226-237, 2008.

9. Soong R, Shah N, Peh BK, et al: The expression of Runx3 in colorectal cancer is associated with disease stage and patient outcome. Br J Cancer 100: 676-679, 2009.

10. Mabuchi M, Kataoka H, Miura Y, et al: Tumor suppressor, AT motif binding factor 1 (ATBF1), translocates to the nucleus with runt domain transcription factor 3 (RUNX3) in response to TGF-beta signal transduction. Biochem Biophys Res Commun 398: 321-325, 2010.

11. Wei D, Gong W, Oh SC, et al: Loss of RUNX3 expression significantly affects the clinical outcome of gastric cancer patients and its restoration causes drastic suppression of tumor growth and metastasis. Cancer Res 65: 4809-4816, 2005.

12. Li WQ, Pan KF, Zhang Y, et al: Relationship between Runx3 expression and precancerous gastric lesions in high-risk population. Beijing Da Xue Xue Bao 41: 348-352, 2009.

13. Li QL, Ito K, Sakakura C, et al: Causal relationship between the loss of Runx3 expression and gastric cancer. Cell 109: 113-124, 2002.

14. Li WQ, Pan KF, Zhang Y, et al: RUNX3 methylation and expression associated with advanced precancerous gastric lesions in a Chinese population. Carcinogenesis 32: 406-410, 2011.

15. Yano $\mathrm{T}$, Ito $\mathrm{K}$, Fukamachi $\mathrm{H}$, et al: The RUNX3 tumor suppressor upregulates Bim in gastric epithelial cells undergoing transforming growth factor beta-induced apoptosis. Mol Cell Biol 26: 4474-4488, 2006.

16. Ito K, Liu Q, Salto-Tellez M, et al: RUNX3, a novel tumor suppressor, is frequently inactivated in gastric cancer by protein mislocalization. Cancer Res 65: 7743-7750, 2005.

17. Nakanishi Y, Shiraha H, Nishina SI, et al: Loss of runt-related transcription factor 3 expression leads hepatocellular carcinoma cells to escape apoptosis. BMC Cancer 11: 3, 2011. 
18. Subramaniam MM, Chan JY, Yeoh KG, Quek T, Ito K and Salto-Tellez M: Molecular pathology of RUNX3 in human carcinogenesis. Biochim Biophys Acta 1796: 315-331, 2009.

19. Ogasawara N, Tsukamoto $\mathrm{T}$, Mizoshita $\mathrm{T}$, et al: RUNX3 expression correlates with chief cell differentiation in human gastric cancers. Histol Histopathol 24: 31-40, 2009.

20. Peng Z, Wei D, Wang L, et al: RUNX3 inhibits the expression of vascular endothelial growth factor and reduces the angiogenesis, growth, and metastasis of human gastric cancer. Clin Cancer Res 12: 6386-6394, 2006.

21. Chang TL, Ito K, Ko TK, et al: Claudin-1 has tumor suppressive activity and is a direct target of RUNX3 in gastric epithelial cells. Gastroenterology 138: 255-265, 2010

22. Hsu PI, Hsieh HL, Lee J, et al: Loss of RUNX3 expression correlates with differentiation, nodal metastasis, and poor prognosis of gastric cancer. Ann Surg Oncol 16: 1686-1694, 2009.

23. Xu HW, Ren F, Chen W, Wang YJ: The influence of survival analysis on RUNX3 gene expression in the primary tumor of patients suffering from stomach carcinoma. Cancer Research on Prevention and Treatment, 2011 (E-pub ahead of print).
24. Ikeda Y, Saku M, Kishihara F and Maehara Y: Effective follow-up for recurrence or a second primary cancer in patients with early gastric cancer. Br J Surg 92: 235-239, 2005.

25. Furukawa $\mathrm{H}$, Imamura $\mathrm{H}$ and Kodera $\mathrm{Y}$ : The role of surgery in the current treatment of gastric carcinoma. Gastric Cancer 5: 13-16, 2002.

26. Zhang XP, Wang ZL, Tang L, Sun YS, Cao K and Gao Y: Support vector machine model for diagnosis of lymph node metastasis in gastric cancer with multidetector computed tomography: a preliminary study. BMC Cancer 11: 10, 2011.

27. Tajima Y, Murakami M, Yamazaki K, et al: Risk factors for lymph node metastasis from gastric cancers with submucosal invasion. Ann Surg Oncol 17: 1597-604, 2010.

28. Park TJ, Han SU, Cho YK, Paik WK, Kim YB and Lim IK: Methylation of O6-methylguaine-DNA methyltransferase gene is associated significantly with K-ras mutation, lymph node invasion, tumor staging, and disease free survival in patients with gastric carcinoma. Cancer 92: 2760-2768, 2001

29. Chen Y, Wei X, Guo C, et al: Runx3 suppresses gastric cancer metastasis through inactivation of MM9 by up-regulation of TIMP-1. Int J Cancer: Dec, 2010 (E-pub ahead of print). 